\title{
La investigación-acción en didáctica de las ciencias: perspectiva desde las revistas españolas de educación
}

\section{Action research in the field of science education: a view from spanish journals on education}

María Jesús Romera Iruela

Departamento de Teoría e Historia de la Educación, Universidad Complutense, Madrid mjromera@edu.ucm.es

RESUMEN • En este artículo se elabora un marco conceptual de la investigación-acción en educación y se ofrece el desarrollo que dicha modalidad de investigación ha tenido en la Didáctica de las Ciencias, a través de la identificación de los artículos publicados en las revistas de educación españolas y del análisis de sus contenidos. Se aporta el perfil personal, social y cognitivo de esta línea de investigación y la incidencia que ha tenido la investigación-acción española en la Didáctica de las Ciencias, en la práctica docente, en la formación del profesorado y en su desarrollo profesional.

PALABRAS CLAVE: investigación-acción; Didáctica de las Ciencias; formación de profesores; práctica docente; enfoque documental.

ABSTRACT • This article presents a conceptual framework for action research in Education. It also provides a detailed analysis of the development this modality of research has revealed in Science Education. The investigation has been performed through the identification of those articles published in Spanish journals of Education and the subsequent detailed analysis of their contents. The personal, social and cognitive profile of this line of research is also analyzed, as well as the impact it has had in Spanish action research in Science Education, in teaching practice, in teacher training and in the professional achievements of those teachers.

KEYWORDS: action research; Science Education; teacher education; teaching practice; documentary approach. 


\section{PLANTEAMIENTO DE LA CUESTIÓN OBJETO DE ESTUDIO}

El estudio de las líneas de investigación en un área científica posibilita el conocimiento de las dimensiones personales, sociales y cognitivas que conforman su propia identidad epistemológica y profesional. Además, como se recogió en el Seminario Internacional sobre «El estado actual de la investigación en la enseńanza de las ciencias», mantener vivo el espíritu de revisión crítica de la investigación es la mejor manera de seguir avanzando en ese -y en cualquier-campo, así como de repercutir de forma creciente y positiva en las practicas educativas (Oliva, 2006: 171). Una de las líneas de investigación que conforman el paradigma de la investigación del profesor o, en sentido más amplio, de la investigación del práctico es la investigación-acción. Ella está presente en la Didáctica de las Ciencias Experimentales, al igual que en la Pedagogía, siendo valorada por su potencialidad de salvar la distancia entre la teoría y la práctica. Al ser un enfoque centrado en el cambio valioso y en la mejora continua de la educación ha de ser prioritario en el quehacer de los profesionales de la enseńanza. Mediante ella se pueden establecer vínculos entre la formación del profesorado, su desarrollo profesional y las necesidades escolares.

A pesar de la relevancia de esta línea de investigación, no contamos con trabajos españoles de síntesis en el campo de la educación (Colas, 200) y las escasas revisiones existentes (Roth, 2008; Zeichner, 2005; Zeichner y Noffke, 2001) han manifestado la necesidad de ampliar su cobertura documental. Nuestro trabajo intenta ser una aportación al respecto, al investigar qué desarrollo tiene la investigación-acción en Didáctica de las Ciencias Experimentales a través de las revistas españolas de educación.

Las cuestiones objeto de estudio, en un nivel documental global, han sido las siguientes:

1. ¿Cuántos artículos se han publicado en las revistas españolas sobre la investigación acción en Didáctica de las Ciencias y cuál ha sido su evolución en el tiempo? ¿En qué medida se han difundido trabajos extranjeros en las revistas españolas?

2. ¿Quienes son los autores más productivos en dicha línea de investigación? ¿Qué grado de colaboración existe entre los autores? ¿Qué grupos de investigación pueden identificarse?

3. ¿Hay colaboración entre instituciones en la realización de esta investigación? ¿Cuáles son las principales entidades en la producción de esta?

4. ¿Cuáles son las principales revistas en la difusión de los artículos sobre el citado enfoque?

5. ¿Qué tipos de artículos se han publicado? ¿Sobre qué temas se ha investigado más? ¿Qué ciencias o materias transversales han concentrado más investigación-acción? ¿Qué grado de atención se ha prestado a los diferentes niveles educativos? ¿Cuáles son los principales paradigmas que orientan dicha investigación?

En un nivel específico, constituido por los artículos españoles que han adoptado el enfoque de la investigación-acción, se dará respuesta a estos interrogantes:

6. ¿Cuáles son los principales objetos de estudio en los artículos españoles de investigación-acción? ¿En qué líneas de investigación de la Didáctica de las Ciencias Experimentales se inscriben?

7. ¿Cuáles son los roles de los investigadores españoles dentro de la función docente?

8. ¿Dicha investigación-acción incide en la práctica docente y en el desarrollo profesional?

A continuación situamos nuestro trabajo en el contexto de los estudios documentales previos y lo inscribimos en el marco de la investigación-acción educativa. 


\section{ANTECEDENTES Y ESTADO DE LA CUESTIÓN}

El interés por el estudio de la investigación del campo de la Didáctica de las Ciencias ha sido continuo desde mediados de la década de los 80 del pasado siglo. Sin pretender ser exhaustivos, vamos a señalar algunos de los trabajos documentales sobre la producción investigadora en dicho campo. En el inicio están las publicaciones de Mata y Méndez (1985), Mata y Anta (1985), Furió y Martínez (1985), De la Gandara (1992) y Palacios y Ansoleaga (1994). La última ha puesto de relieve que, en los trabajos estudiados sobre investigación e innovación en la enseñanza, un 14\% de ellos eran de investigación-acción. Al cumplir una década de publicación la revista Enseñanza de las ciencias, se realizaron los trabajos de Gil (1994) y Moreira (1994). Entre los más actuales, mencionamos los de Elórtegui, Fernández y Medina (2002), Anta y Pérez (2007), Anta (2008) y De Pro (2009). Además, no podemos dejar de citar algunos de los presentados en los Encuentros de Didáctica de las Ciencias Experimentales, entre ellos, los de García Barros (2008), Benarroch (2010) y Oliva (2010). Más allá de nuestro país, destacan los análisis, en continuidad, realizados por Tsai y Wen (2005) y Lee, Wu y Tsai (2009). Ninguno de los trabajos previos se ha centrado las publicaciones sobre investigación-acción. El nuestro dará a conocer la evolución de esa línea de investigación en la Didáctica de las Ciencias Experimentales desde las revistas españolas.

La investigación-acción se ha configurado, siguiendo a Zeichner (2005), desde la actividad de cinco grandes tradiciones. La primera es la tradición de la investigación-acción en Estados Unidos. En ella destaca Corey, profesor en la Universidad de Columbia, que se nutrió del trabajo de Lewin. Este profesor pensaba que dicha investigación podía mejorar y cambiar la práctica del currículum, dado que los profesionales utilizarían los resultados de sus propias investigaciones. Entendía, igual que aquel, que la investigación-acción era un proceso cíclico en el que cada ciclo afectaba a los siguientes y perfiló las fases de dicho proceso. El desarrollo de grandes proyectos en las escuelas dio lugar a la llamada «era de la investigación-acción cooperativa». Al final de la década de los cincuenta del pasado siglo esta investigación se debilita y, prácticamente, desaparece de la literatura educativa hasta los años ochenta, que es cuando aparece el movimiento norteamericano de la investigación del profesor (Corey, 1953; Zeichner, 2005). La segunda tradición está constituida por el movimiento del profesor como investigador, en el Reino Unido, que evolucionó a partir del trabajo de reforma del currículum realizado por los profesores ingleses, en los años 1960 y 1970, y del apoyo prestado por algunos de la universidad, entre ellos, Stenhouse y Elliott. El primero es quien lo impulsa desde su pensamiento de que el profesor es un artista y, como tal, tiene que usar la autonomía de juicio, que está basada en la investigación orientada al perfeccionamiento de su arte. De ahí, que él sea tema de investigación y de desarrollo (Stenhouse, 1985: 49). Elliott participó en la realización de estudios de casos que utilizaban la investigación-acción de segundo orden para explorar cómo se puede facilitar el aprendizaje profesional de los docentes a través de esa misma metodología. En 1978 publicó un artículo titulado «¿Qué es la investigación acción en las escuelas?». Se trata de un proceso que gira en torno a sucesivos ciclos de reflexión y de acción (Elliott, 1993). De ahí que la práctica reflexiva sea la forma de la investigación educativa y de la intervención en la formación de los profesores. El trabajo de estos y muchos otros profesionales ha llevado a la creación de la red CARN (Red de Investigación Acción Colaborativa). La tercera tradición, que tiene influencia de la anterior, es la investigación-acción participativa australiana, que se inscribe en el marco de la Teoría Crítica de la Escuela de Frankfurt y, en particular, en la epistemología de Habermas. Su principal representante en el ámbito de la educación es Kemmis quien, con la colaboración de otros profesionales de la Universidad de Deakin, ha elaborado un modelo emancipatorio que, con forma de espiral cíclica, tiene cuatro fases: planificación, acción, observación y reflexión (Kemmis y McTaggart, 1992). El citado movimiento norteamericano de la investigación del profesor es la cuarta tradición que, tras un número de años de investigación y desarrollo interactivos y de otras formas de investigación co- 
laborativa en educación, se ha ido desarrollando, desde 1980, sobre todo por profesores, con apoyo de sus colegas de la universidad y de las asociaciones de sus materias. Se han formado, también, comunidades gestionadas por los profesores. La última tradición es la de la investigación de autoestudio, que está emergiendo, con gran aceptación, desde la década de 1990. En ella se utiliza la investigación-acción para estudiar la enseńanza en universidades e instituciones de educación superior. Se está utilizando mucho en la formación del profesorado. En la Asociación Americana de Investigación Educativa existe un grupo de «auto-estudio de las prácticas de formación de profesores» (Zeichner, 2005: 276).

La investigación-acción se ha realizado desde los distintos paradigmas existentes en el campo de la educación. Aunque no es fácil definir este enfoque, compartimos la delimitación que hacen del mismo Reason y Bradbury (2005): el término investigación-acción incluye toda la familia de enfoques de investigación que son participativos, basados en la experiencia, y porque están orientados a la acción, hablando en términos prácticos, son generalmente reconocibles y no "propiedad» exclusiva de una tradición (p. xxiv).

Shafer (2000) ha descrito la investigación acción como una de las tres formas que constituyen el continuo de la investigación del profesor, situándola en el centro entre la práctica reflexiva y la investigación cualitativa. La investigación del profesor está adquiriendo un notable desarrollo y, en el ámbito de la enseñanza de las ciencias, se le ha dedicado un capítulo en el último tratado de investigación sobre educación científica -Handbook of Research on Science Education (2008)-. Los enfoques de la investigación-acción y de la investigación del profesor adquieren una mayor actividad y visibilidad en la comunidad de la Educación en Ciencias en la década de los noventa del pasado siglo, cuando algunos másteres de formación del profesorado y programas de desarrollo profesional empezaron a requerir que sus destinatarios realizaran investigaciones en su práctica, si bien, probablemente, son múltiples los acontecimientos que han contribuido a este movimiento (Roth, 2008).

La reciente revisión sobre los profesores de ciencias como investigadores (Roth, 2008) recoge el estado actual de la investigación-acción en la educación científica. Ella pone de manifiesto que la investigación acerca del aprendizaje del profesor y la referida a la relación entre investigación y práctica sugieren dos roles para la investigación del profesor de ciencias: apoyar el aprendizaje del profesor así como su crecimiento profesional y contribuir a la base de conocimientos sobre la enseńanza y el aprendizaje de las ciencias, enlazando investigación y práctica. La investigación-acción ha sido utilizada en los programas de formación inicial, en los de perfeccionamiento y en los de desarrollo profesional de los profesores de ciencias emprendidos por universidades y otras entidades externas al ámbito escolar. Proyectos con esa misma metodología han sido iniciados y apoyados por las escuelas y por los distritos escolares. El trabajo citado, al analizarlos, ha puesto de manifiesto que existe amplio acuerdo, en la comunidad de Educación en Ciencias, en la idea, inicialmente expresada por Stenhouse, de que la enseñanza debería ser una forma de indagación, experimentación o investigación. Además, hay evidencia de que la investigación-acción y la del profesor de ciencias contribuyen a la preparación profesional, al aprendizaje y al crecimiento del profesorado, si bien existe una menor evidencia de que ellas afectan a la práctica del profesorado de ciencias en formas que conducen a una mejora del aprendizaje del alumno. Asimismo, aunque hay dificultades en dichas investigaciones, ellas ofrecen una perspectiva interna única que podría ayudar a cerrar la brecha señalada entre la investigación académica tradicional y la práctica de la enseñanza de las ciencias. No obstante, es preciso señalar que las referidas investigaciones están en su infancia y que el debate sobre su eficacia y utilidad está presente (p.1246). La controversia se centra en los estándares de calidad con los que hay que juzgar esas investigaciones y en el tipo de conocimiento que ellas generan (Zeichner y Noffke, 2001).

Entre los autores españoles, Furió (1994) ha señalado la necesidad de integrar la investigación e innovación educativa, como actitud, en la formación y reciclaje de los profesores de ciencias, basándose en programas y recomendaciones que indisolublemente han asociado la docencia y la investigación. 
Previamente Sáez (1987) había señalado la relevancia de la investigación-acción en la formación del profesorado español. En el ámbito de Didáctica de la Física, García-Carmona (2009) ha expresado que dicha investigación es el instrumento idóneo en el control y la regulación del proceso de enseñanza y aprendizaje, así como la metodología propicia para el auto-desarrollo profesional del profesorado. En los congresos internacionales sobre Investigación en Didáctica de las Ciencias hemos identificado 26 trabajos en los que se presentan proyectos o experiencias de investigación acción en la formación de ese profesorado y en la enseñanza y el aprendizaje de las ciencias.

Antes de informar sobre el estado de esta investigación en las revistas españolas, exponemos el método con el que hemos procedido.

\section{METODOLOGÍA}

Nuestra investigación se ha realizado siguiendo un diseño de examen y procediendo con una metodología documental. Para la identificación de los artículos se han consultado las principales bases de datos sobre educación. El proceso secuencial y el procedimiento es el que describimos a continuación. Inicialmente acudimos a las bases ISOC-Educación y REDINED. La interrogación de las mismas se realizó utilizando su lenguaje documental, Tesauro Europeo de la Educación, y el lenguaje natural. En el cuadro 1 esquematizamos las cadenas de la búsqueda con descriptores, que ascienden a $34(33+1)$. Todas, excepto la última, están formadas por tres descriptores (dos de la primera columna unidos con cada uno de los de la segunda por el operador «y»). A su vez, los resultados obtenidos en las 34 búsquedas fueron combinados, aditivamente, mediante el operador booleano «o» $\mathrm{y}$, posteriormente, con los hallados en las interrogaciones con los dos lenguajes.

Cuadro1.

Cadenas de descriptores de la búsqueda en lenguaje documental

\begin{tabular}{|l|l|}
\hline Descriptores 1 y 2: & Descriptor 3 \\
\hline & Ciencias de la naturaleza \\
& Ciencias biológicas \\
Biología & Ciencias físicas \\
a) Investigación-acción y didáctica y & Física \\
\hline b) Investigación-acción y aprendizaje y & Química \\
\hline C) Investigación acción y enseñanza y & Geología la tierra \\
& Ecología \\
& $\begin{array}{l}\text { Medio ambiente } \\
\text { Salud }\end{array}$ \\
\hline d) Investigación acción y educación científica. & \\
\hline
\end{tabular}

Los perfiles de búsqueda complementarios, en ambos lenguajes, están delineados en el cuadro 2 . De nuevo, los descriptores de la primera columna de todas las filas se combinaron, mediante el operador «y», con todos los descriptores y no descriptores que figuran en las columnas segunda y tercera de las mismas filas. A continuación, se combinaron, aditivamente («0»), los resultados de las búsquedas de todas las cadenas. 
Cuadro 2.

Cadenas de descriptores y no descriptores de las búsquedas complementarias

\begin{tabular}{|c|c|c|}
\hline Descriptores & Descriptores & No descriptores \\
\hline e) Investigación acción y & & $\begin{array}{l}\text { - Enseñanza de las ciencias } \\
\text { - Ciencias medioambientales } \\
\text { - Medio natural }\end{array}$ \\
\hline $\begin{array}{l}\text { f) Didáctica } \\
\text { g) Enseñanza } \\
\text { h) Aprendizaje }\end{array}$ & $\begin{array}{l}\text { - Ciencias de la naturaleza } \\
\text { - Ciencias biológicas } \\
\text { - Biología } \\
\text { - Ciencias físicas } \\
\text { - Física } \\
\text { - Química } \\
\text { - Ciencias de la tierra } \\
\text { - Geología } \\
\text { - Ecología } \\
\text { - Medio ambiente } \\
\text { - Salud }\end{array}$ & $\begin{array}{l}\text { - Investigación colaborativa } \\
\text { - Investigación cooperativa } \\
\text { - Investigación participativa }\end{array}$ \\
\hline \multirow[t]{2}{*}{ i) Educación científica } & & $\begin{array}{l}\text { - Investigación colaborativa } \\
\text { - Investigación cooperativa } \\
\text { - Investigación participativa }\end{array}$ \\
\hline & $\begin{array}{l}\text { No descriptores } \\
\text { j) Investigación colaborativa } \\
\text { k) Investigación cooperativa } \\
\text { 1) Investigación participativa }\end{array}$ & $\begin{array}{l}\text { - Enseńanza de las ciencias } \\
\text { - Ciencias medioambientales } \\
\text { - Medio natural }\end{array}$ \\
\hline
\end{tabular}

El sentido del término investigación-acción, que recoge el mencionado tesauro en una nota de alcance, es el siguiente: «Método de investigación realizado por los participantes en procesos sociales con objeto de mejorar la racionalidad de sus propias prácticas sociales, el entendimiento de las mismas y las situaciones en las que se llevan a cabo» (p. 216).

$\mathrm{Al}$ objeto de obtener la mayor exhaustividad en la cobertura documental consultamos, con los perfiles descritos, otras dos bases de datos: Compludoc y Dialnet. La recuperación finalizo con la consulta de Google Académico. El periodo temporal abarcado es desde 1976 (primero de la base ISOC) a 2010 (mediados de junio), si bien conviene tener en cuenta la distancia existente entre la publicación de las revistas y su indización en las bases de datos.

Posteriormente, efectuamos un proceso de revisión de las referencias obtenidas y eliminamos los solapamientos entre las fuentes, así como otra tipología documental asociada en las bases que no eran artículos de revistas. Una vez efectuada la selección de las referencias, creamos, a través de Refworks, la correspondiente base de datos. A continuación, aplicamos la técnica del análisis de contenido a las dimensiones recogidas en la cuestión objeto de estudio. En el establecimiento de las categorías hemos tenido en cuenta las de otros trabajos previos próximos al nuestro (De la Gandara, 1992; Oliva, 2006; Palacios y Ansoleaga, 1993, y Tsai, 2005, entre otros), si bien hemos procedido por vía inductiva, excepto en las de los paradigmas y líneas de investigación, en donde hemos partido de los enfoques consolidados y las líneas más habituales. Finalmente, realizamos el análisis de los datos y su interpretación. 


\section{RESULTADOS}

Siguiendo las cuestiones del problema objeto de investigación damos cuenta de los resultados obtenidos.

\section{Producción de artículos sobre investigación acción en Didáctica de las Ciencias}

A través del proceso de recuperación documental que acabamos de describir hemos obtenido un total de 83 artículos sobre investigación-acción en Didáctica de las Ciencias Experimentales. La distribución cronológica de los mismos se presenta en el gráfico 1.

\section{Gráfico 1.}

Produccion de artículos sobre investigación-acción en Didactica de las Ciencias Experimentales

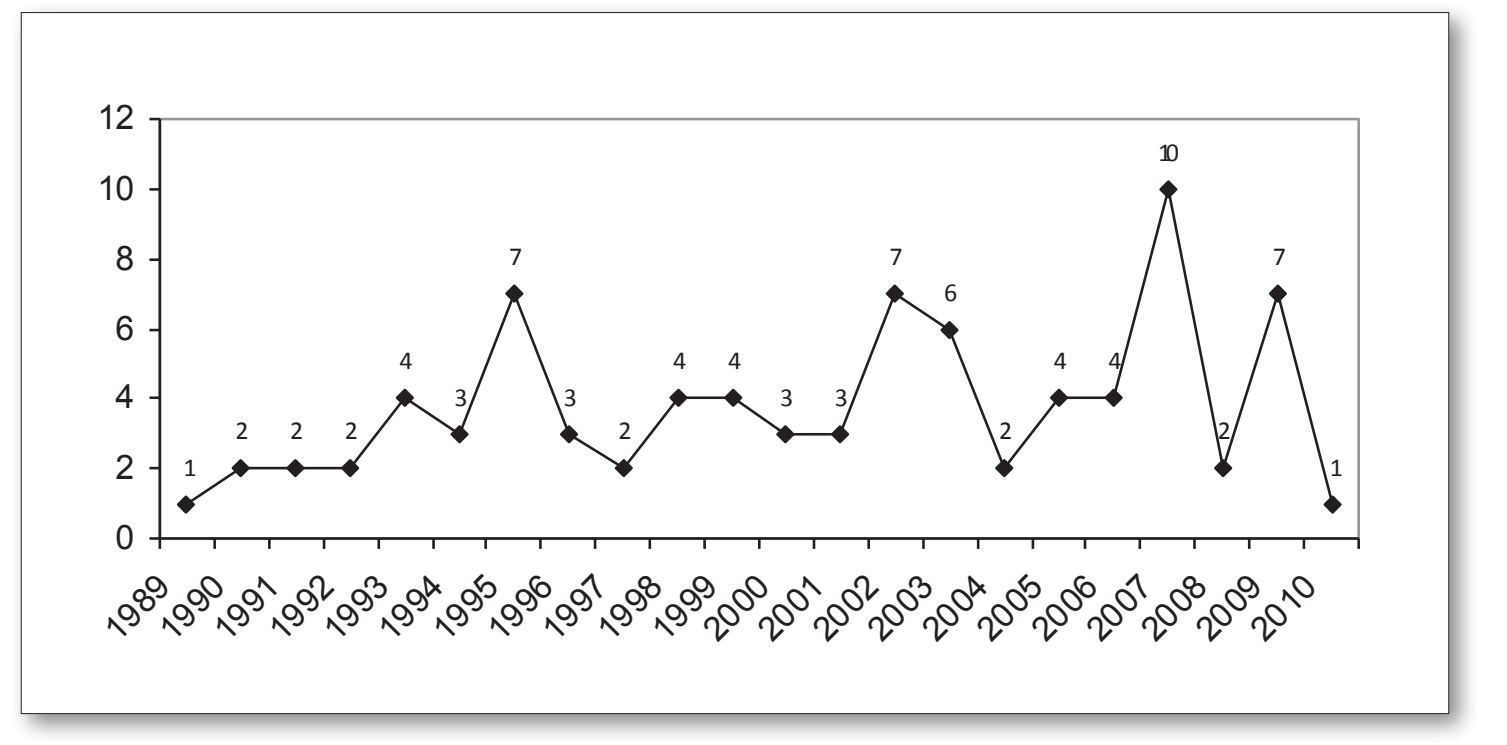

El primer artículo encontrado sobre el tema se publicó en el año 1989, siendo el único en ese año. En el otro extremo, en el 2010, tan solo se ha recuperado un artículo, dato que se puede explicar por el momento en el que se ha cerrado la búsqueda y por el mencionado lapso temporal entre la edición de las revistas y su indización. La fecha de inicio coincide con el momento en el que se estaba gestando la reforma educativa de la LOGSE, que iba a promover esta modalidad de investigación. Si nos centramos en el periodo de 22 ańos en el que existen artículos, se aprecia la existencia de una producción continua, pero baja, siendo la media anual de 3,8. El mayor número de artículos, que asciende a 10, lo encontramos en el año 2007. La aportación de cuatro trabajos por un grupo de autores proporciona una cierta explicación de ese incremento. Existen otros tres momentos, 1995, 2002 y 2009, en los que se concentran 7 artículos, que es la siguiente cantidad más alta. En el primero y, sobre todo, en el segundo de esos momentos hay incidencia, con 2 y 4 artículos, de dos monográficos cuyos temas fueron ¿Cómo enseñar? e Investigación e innovación en la enseñanza de las ciencias.

Del total de artículos publicados en las revistas españolas que hemos recuperado, un 71,1\% de ellos han sido realizado por autores españoles, un $26,5 \%$ por personas de otras nacionalidades y un $2,4 \%$ por profesionales españoles en colaboración con otros, en un caso, de Argentina y, en el otro, de Cuba. Los artículos extranjeros proceden de 9 países, siendo Argentina el que aporta el mayor número (9), seguida por Venezuela (4), Brasil (2), Italia y Portugal (2). Australia, México, Reino Unido y Turquía contribuyen con un trabajo. 


\section{Autores más prolíficos, colaboración y grupos de investigación}

En la elaboración de los 83 artículos identificados han participado 128 autores, siendo uno de ellos un colectivo. El estudio de esta productividad nos ha permitido identificar a los autores más prolíficos en esta línea de investigación. En la tabla 1 se han incluido aquellos que han publicado más de tres trabajos.

Tabla 1.

Autores más productivos en artículos sobre investigación-acción en Didáctica de las Ciencias

\begin{tabular}{|c|c|l|}
\hline Número de artículos & Número de autores & \multicolumn{1}{|c|}{ Identidad de los autores } \\
\hline 7 & 1 & P. Membriela Iglesia \\
\hline 6 & 1 & R. Jiménez Pérez \\
\hline 5 & 2 & $\begin{array}{l}\text { V. Mellado Jiménez } \\
\text { B. Vázquez Bernal }\end{array}$ \\
\hline 4 & 3 & $\begin{array}{l}\text { A. Lacueva } \\
\text { M. M. Martínez Aznar } \\
\text { M. P. Varela Nieto }\end{array}$ \\
\hline
\end{tabular}

No existen grandes productores en esta perspectiva temático-metodológica ya que el máximo productor, P. Membriela Iglesia, ha publicado un total de 7 artículos en el periodo estudiado. El número de autores que han participado en al menos 3 trabajos asciende solo a 8. Esto nos indica que, a pesar de la relevancia de esta modalidad de investigación, hay un cultivo muy minoritario, al menos en realizaciones comunicadas formalmente a través de las revistas científicas. Si a ello unimos que son pocos los trabajos que hemos encontrado en las actas del principal congreso del Área, podemos concluir, en la línea de la literatura internacional, que estamos al comienzo de la infancia de la investigación-acción en Didáctica de las Ciencias Experimentales.

El grado de colaboración entre los autores de los artículos se ha calculado mediante el índice de firmas/trabajo. En el conjunto de los artículos hay 170 firmas. El 41\% de ellos tienen una sola firma, el $27,7 \%$ dos y el 31,3\% tres o más. El índice de colaboración en la línea de investigación es 2,05. Dicho valor es igual al establecido para las ciencias sociales en España (2 firmas por trabajo) y superior a los encontrados en los artículos españoles de Didáctica de las Ciencias y de Educación Matemática (1,81 y 1,84), así como al estimado en la investigación educativa, inferior a 2 (Anta y Pérez, 2007; Maz y otros, 2009). Dicha superioridad se debe a la propia naturaleza participativa de la investigación acción.

En la identificación de los grupos de investigación, a través de la publicación, hemos seguido la técnica sugerida por Price y Beaver (1966), y luego generalizada por Peiró y sus colaboradores (1981), de acuerdo con la cual un grupo queda constituido por aquellos autores que colaboran en las investigaciones y por todos los colaboradores de cada uno de estos autores. Adoptando como criterio mínimo que cada grupo incluya, por lo menos, tres autores y más de tres artículos, hemos encontrado tres de esos grupos.

El primero de ellos lo forman 5 autores, siendo P. Membriela Iglesia el principal. Aportan, en su conjunto, nueve artículos. Tres de ellos los ha escrito el autor citado solo; en otros dos hay colaboración con M. Suárez Pazos, y en uno de estos participa también E. Nogueiras. A su vez, la primera colaboradora elabora otro de estos documentos. M.D. Dapía Conde publica un trabajo con P. Membriela Iglesia y M.C. Cid Manzano; los dos últimos autores presentan otro en común, mientras que la primera firma sola un segundo artículo. La temática de estas investigaciones es el diseño de proyectos 
curriculares innovadores de ciencias y la formación del profesorado del área. Los componentes de este grupo han ejercido su profesión en las siguientes instituciones: Escuela Universitaria de Formación del Profesorado, Facultad de Humanidades y Facultad de Ciencias de la Educación de la Universidad de Vigo y en el Instituto de Bachillerato Otero Pelayo de Orense.

El segundo grupo está compuesto por 6 personas que realizan seis trabajos. Está articulado en torno a B. Vázquez Bernal, quien colabora en cinco artículos con R. Jiménez Pérez y V. Mellado Jiménez; en dos de ellos también figura C. Taboada Leñero y en otro distinto aparece M. Martos Carrasco. Previamente, R. Jiménez Pérez había publicado una investigación con A.M. Wamba Agudo. Su línea de investigación es la formación y enseñanza de las ciencias desde el modelo de práctica reflexiva. Las instituciones en las que trabajan son: el Departamento de Didáctica de las Ciencias y Filosofía de la Universidad de Huelva, el de Didáctica de las Ciencias Experimentales y Matemáticas de la Universidad de Extremadura, el IES Jorge Juan en San Fernando (Cádiz ), el IES Diego Macias en Calañas (Huelva) y otro IES de Málaga.

El tercer y último grupo reúne a 6 autores que proporcionan 5 artículos. La autora principal es $\mathrm{M}$. M. Martínez Aznar quien colabora en tres publicaciones con M. P. Varela Nieto y en otra con M. T. Ibánez Orcajo. Con anterioridad, M. C. Pérez-Lanzábal produjo un trabajo con A. Favieres Martínez, M. J. Manrique del Campo y M. P. Varela Nieto. La resolución de problemas en Física y, en menor medida, en Genética es la temática que han tratado. Desempeñan su actividad en las siguientes entidades: Instituto de Electrónica de las Comunicaciones del CSIC, Departamento de Didáctica de las Ciencias Experimentales de la Universidad Complutense de Madrid, Instituto de Bachillerato Mariana Pineda, Instituto Rey Pastor, Instituto Ramiro de Maéztu e I ES Meco, que están en la ciudad mencionada previamente.

\section{Colaboración institucional y principales entidades en la realización de esta investigación}

El análisis de la afiliación institucional de los autores permite determinar si hay colaboración entre diferentes entidades en la realización de los trabajos y cuáles son las más especializadas en investigaciónacción. En un $67,5 \%$ de los artículos consta una institución, en un 19,3\% de ellos, dos, en un 9,6\%, tres, y en un 3,6\%, cuatro. Por tanto, la colaboración interinstitucional se produce en el 32,5\% de los trabajos. Su mayor grado se da entre las universidades. Le sigue la de aquellas con los institutos de enseñanza secundaria y, a continuación, la de las primeras con los centros de educación infantil y primaria. Junto a ellos, hay otros organismos cuya presencia es mínima.

En la universidad se ha realizado el 81,9\% de los artículos; el 18\% restante en 11 entidades, predominando los institutos de enseñanza secundaria y colegios en los que se imparten diferentes niveles educativos. El número total de universidades asciende a 37, de las que 13 son españolas y 24 extranjeras. Entre las primeras destaca, en primer lugar, la Universidad de Vigo, en la que se han realizado 9 artículos. Le siguen las de Extremadura, Huelva y Sevilla, con 6 trabajos, y luego la Universidad Complutense, con 5, y la Universidad Autónoma de Barcelona, con 4. En relación con las universidades extranjeras, 4 artículos proceden de la Universidad Central de Venezuela y en el resto solo se han realizado uno o dos.

\section{Revistas más relevantes en la difusión de esta investigación}

Los artículos sobre investigación-acción en Didáctica de las Ciencias han sido publicados en 28 títulos de revistas. Los primeros rangos en la comunicación de esta investigación los ocupan Enseñanza de las Ciencias (19 trabajos) y Alambique (15). Mediante ellas se pueden recuperar el 41\% de los artículos. La tercera revista, en grado de difusión, es Investigación en la Escuela (8). En las dos siguientes posiciones 
se encuentran Enseñanza de las Ciencias de la Tierra y Revista Electrónica de Enseñanza de las Ciencias, con cinco artículos.

La distribución de los artículos en las revistas sigue la ley de Bradford. Un número importante de artículos se concentran en pocas revistas mientras que el resto se dispersan en un gran número de ellas. Con tan solo tres revistas se pueden obtener el $50 \%$ de los trabajos.

\section{Naturaleza de los artículos y temáticas tratadas}

En la determinación del tipo de trabajos recogidos en los artículos sobre investigación-acción en Didáctica de las Ciencias Experimentales hemos identificado, inductivamente, dos categorías: estudios descriptivos y reflexivos, a los que denominamos «artículos teóricos», y experiencias o proyectos de investigación sobre la temática estudiada. Un $21,7 \%$ de los artículos son del primer tipo, mientras que un $78,3 \%$ lo son del segundo.

En cuanto a las temáticas de los artículos, un $49,4 \%$ de estos se refieren a la enseñanza de las ciencias, un 22,9\% a la formación del propio profesorado, un 20,5\% al aprendizaje de las ciencias, un 6\% a cuestiones de epistemología y un 1,2\% a la perspectiva del género. Algunas de estas categorías presentan subdivisiones. La primera contiene tres aspectos: diseño, desarrollo y evaluación del currículum $(33,7 \%)$, estrategias y métodos de instrucción $(12,1 \%)$ y pensamiento del profesor $(3,6 \%)$. La tercera incluye los siguientes contenidos referidos al citado aprendizaje: contextos de clase (laboratorio, trabajos prácticos, lenguaje, discurso: 7,2\%), concepciones de los estudiantes y cambio conceptual (6,1\%), evaluación $(3,6 \%)$, aprendizaje no formal e informal de las ciencias $(2,4 \%)$ y actitudes $(1,2 \%)$. Estas temáticas presentan una amplia coincidencia con los principales focos de la investigación actual en la enseñanza de las ciencias y con sus prioridades, en cuanto a líneas a emprender o a continuar (Oliva, 2006).

Desde el punto de vista de las disciplinas científicas y de los temas transversales relacionados con ellas, hemos encontrado que el 25,3\% de los artículos se centran en las ciencias, en general, o en las ciencias experimentales en conjunto, un $15,7 \%$ de ellos son investigaciones sobre Física, a ellos hay que añadir un 2,4\% que versan sobre dicha ciencia junto con la Química, un 14,5\% se refieren a la Educación ambiental, un 13,3\% aluden a las Ciencias Naturales o a los enfoques, en ellas, de Ciencia, Tecnología y Sociedad, un 7,2\% se ocupan de la Geología y las Ciencias de la tierra, un 6\% se refieren al Conocimiento del medio, un 4.8\% consideran la Educación para la salud, un 3,6\% atienden a la Biología y las Ciencias Biológicas, un 2,4\% estudian cuestiones de Química, junto con el mismo porcentaje previo que es común con la Física, otro 2,4\% sostiene la interdisciplinariedad, un 1,2\% trata sobre Ecología y, finalmente, un porcentaje igual alude a las Ciencias Morfológicas. Existe convergencia con los resultados de los trabajos previos en cuanto al predominio de la investigación en Física, Educación ambiental/Ciencias medioambientales y Ciencias Naturales, pero, en nuestros datos, hay un descenso en la Química (Anta y Pérez, 2007; Mata y Méndez, 1985; Palacios y Ansoleaga, 1993).

\section{Atención concedida en los artículos a los distintos niveles del sistema educativo}

En orden a conocer el grado de atención prestada a los distintos niveles del sistema educativo, realizamos una nueva categorización y clasificación de los artículos. Los resultados de la misma se presentan en la tabla 2. El análisis de los datos pone de manifiesto que la mayor parte de estas publicaciones se han situado en la Educación Secundaria. Después, el interés se ha centrado en la Educación Primaria, cuyo valor supera al de la Educación Universitaria, si tenemos presentes sus vínculos, sobre todo con la Educación Secundaria. La investigación-acción apenas ha incidido en Educación Infantil. Estos resultados apoyan los de los trabajos previos sobre la investigación en Didáctica de las Ciencias, los cuales 
han indicado que el foco de atención de dicha investigación se encuentra en la Enseñanza Secundaria, siendo muy escasa en las de Infantil y Primaria (Anta y Pérez, 2007; Benarroch, 2010; De Pro, 1998, 2009; García Barros, 2008; Oliva, 2006, 2010).

Tabla 2.

Distribución de los artículos en función de los niveles de enseñanza

\begin{tabular}{|c|c|c|}
\hline Niveles & Número de artículos & Porcentaje de artículos \\
\hline Infantil & 1 & $1,2 \%$ \\
\hline Infantil y Primaria & 2 & $2,4 \%$ \\
\hline Primaria & 10 & $12,1 \%$ \\
\hline Primaria y ESO & 8 & $9,6 \%$ \\
\hline Primaria, ESO y Bachillerato & 2 & $2,4 \%$ \\
\hline $\begin{array}{l}\text { Secundaria: } \\
\text { - ESO } \\
\text { - Bachillerato } \\
\text { - ESO y Bachillerato }\end{array}$ & 36: & $\begin{array}{r}26,5 \% \\
8,4 \% \\
8,4 \%\end{array}$ \\
\hline Universitaria/Superior & 13 & $15,7 \%$ \\
\hline $\begin{array}{l}\text { Más de } 2 \text { niveles: } \\
\text { - Inf., Prim., Sec. y Univ. } \\
\text { - Prim, Sec. y Univ. } \\
\text { - Prim., ESO, Bach. y FP } \\
\text { - Prim, ESO, Bach y COU }\end{array}$ & $\begin{array}{ll}7: & \\
1 \\
3 \\
1 \\
2\end{array}$ & $\begin{array}{r}8,4 \% \\
1,2 \% \\
3,6 \% \\
1,2 \% \\
2,4 \% \\
\end{array}$ \\
\hline No consta u otros & 4 & $4,8 \%$ \\
\hline TOTAL & 83 & $100 \%$ \\
\hline
\end{tabular}

\section{Enfoques epistemológicos en los artículos}

La investigación-acción se ha realizado en el marco de los distintos paradigmas existentes en el ámbito de las ciencias humanas y sociales: empírico-positivista/postpositivista, interpretativo-práctico, críticoemancipatorio, sistémico/de la complejidad y participativo. Dichos enfoques, en su delimitación usual (Guba y Lincoln, 2005; Morin, 1998; Schwandt, 1994), han constituido las categorías de partida. El enfoque epistemológico predominante es el interpretativo-práctico, que está presente en un 49,4\% los artículos, le sigue el crítico-emancipatorio, que consta en un 15,7\% de estos; una combinación de ambos se encuentra en un 9,6\% de ellos y otra interrelación de los dos últimos con el paradigma sistémico/de la complejidad se da en un 10,8\% de los trabajos; este último enfoque está vinculado con el interpretativo en un 2,4\% de los artículos; menor incidencia tiene el paradigma empírico positivista/ postpositivista, que se halla en un 7,2\% de los artículos y en un 1,2\% adicional figura junto con el interpretativo y el crítico. El paradigma participativo no tiene ninguna presencia. Finalmente, en un $3,6 \%$ de estas publicaciones no se menciona ni se ha podido inferir ningún enfoque. El constructivismo aparece unido a todos los paradigmas previos que han orientado los artículos, estando presente en un $61,4 \%$ de ellos.

Los autores pertenecientes a las tradiciones de la investigación-acción educativa que han sido más citados son: Elliott, en un 30,1\% de los artículos; Carr y Kemmis, en un 20,5\%, y Kemmis y McTaggart, en otro 6\%; Stenhouse, en un 16,9\%, y Schön, en un 7,2\% de ellos. 


\section{Principales cuestiones objeto de estudio en los artículos españoles de investigación-acción y líneas de investigación en Didáctica de las Ciencias en las que se inscriben}

En el conjunto de los artículos realizados por autores españoles que contienen experiencias o proyectos de investigación acción $(\mathrm{n}=47)$, hemos examinado las cuestiones objeto de estudio así como las líneas de investigación en las que se inscriben. Las categorías de clasificación de dichas líneas han sido, básicamente, con alguna modificación introducida de forma inductiva, las que se señalan en los trabajos de De Pro (2010), Gil, Carrascosa y Martínez (2000), Fernández, Medina y Elórtegui (2002), Mellado (1998) y Oliva (2006). En la asignación de las cuestiones estudiadas en los artículos a las categorías hemos mantenido la exclusividad, a pesar de que muchas pueden inscribirse en más de una. Debido a los límites de espacio, en el cuadro 3 presentamos solo una muestra de dichos objetos de estudio, problemas o propósitos de las investigaciones, inscritos en sus líneas de investigación.

\section{Cuadro 3.}

Algunos objetos de estudio de los artículos inscritos en las líneas de investigación

Formación del profesorado

- Se investiga el nivel de reflexión de una profesora de ciencias, su evolución en el tiempo y los obstáculos que dificultan la complejidad en la reflexión e impiden un desarrollo deseable.

\section{Resolución de problemas}

- Se investiga hasta qué punto un proceso de enseńanza encaminado a familiarizar a los alumnos de bachillerato con una metodología investigativa de resolución de problemas de física produce un cambio conceptual coherente y duradero.

\section{Educación ambiental}

- Conocer la influencia y eficacia de una estrategia de intervención Proyecto Ecocentros para el desarrollo de la educación ambiental en los centros educativos.

Relación ciencia/tecnología/sociedad y el papel del medio

- Elaboración de dos proyectos curriculares innovadores de ciencias para la enseñanza secundaria, diseñados por un grupo de investigación-acción, cuya meta fundamental es la relevancia social y personal.

Concepciones previas de los estudiantes

- Se investiga acerca de las dificultades experimentadas por los alumnos de $2 .^{\circ}$ de bachillerato al enfrentarse a un problema habitual basado en el teorema de Gauss.

Evaluación

-Evaluar el trabajo de campo realizado por los alumnos, a través de una metodología basada en el modelo de investigación-acción, atendiendo a los conceptos, procedimientos y actitudes.

Diseño y desarrollo curricular en el área de ciencias

- Elaborar y caracterizar una propuesta metodológica basada en la investigación, para los alumnos y profesores, en la que el huerto es el organizador del conocimiento.

Prácticas de laboratorio

- Introducir una innovación en una práctica de Física relativa al segundo principio de la dinámica, remodelándola y convirtiéndola en una investigación.

Lenguaje (comunicación escrita)

- Analizar, desde los criterios diseñados, la redacción de informes de investigación de siete tareas de laboratorio realizadas por alumnos de Bachillerato, valorando si mejoran esos productos.

Alfabetización científica

- Diseñar y difundir una práctica de ciencia cotidiana en la que una preparación culinaria permite la aproximación a la experimentación y a los mecanismos físico-químicos del proceso. 
Los objetos de estudio de los artículos españoles se incardinan en diez líneas de investigación. La que recibe más atención es la formación del profesorado (31,9\%), a la que aportan propuestas dirigidas a cambiar concepciones y prácticas hacia enfoques constructivistas $(6,4 \%)$, enfocadas a capacitar al profesorado como diseñador de proyectos curriculares e investigador de su acción en el aula $(8,5 \%)$ y orientadas a desarrollar prácticas docentes desde el modelo de enseñanza como profesión reflexiva (17\%). La mayoría de ellas se dirigen al profesorado en activo. La resolución de problemas está en la segunda posición $(14,9 \%)$ y mediante dicha metodología se ha estudiado el cambio conceptual y actitudinal en física y, en menor medida, en genética. Seguidamente, con el mismo interés $(12,8 \%)$, se sitúan la educación ambiental, a la que se contribuye con proyectos de integración de la misma en el currículum de ciencias y en los centros educativos, y, vinculada a ella, el enfoque ciencia/tecnología/ sociedad y el papel del medio, con integración de áreas y materias, en la que también se desarrollan proyectos innovadores. Los dos siguientes rangos, con idéntica proporción (8,5\%), son para las líneas evaluación, en la que se han elaborado y aplicado modelos basados en la investigación-acción, y concepciones previas, en la que se ha investigado la conceptualización de la energía y la luz, así como un problema basado en el teorema de Gauss. Aunque solo un 2,1\% de los artículos que presentan propuestas curriculares se han incluido en la línea diseño y desarrollo curricular, hay que advertir que el cultivo de la misma es mayoritario ya que casi todos los trabajos podrían haberse incluido en ella. Además, el porcentaje previo $(2,1 \%)$ lo tienen otras tres líneas: prácticas de laboratorio, lenguaje y alfabetización científica. Lo que se ha indagado en ellas consta en el cuadro 3. Por último, el 2,1\% restante no tiene vinculación con las líneas precedentes y ha estudiado los aspectos psicopedagógicos del aprendizaje de circuitos eléctricos.

\section{Roles de los investigadores españoles dentro de la función docente}

El análisis de los roles de los investigadores dentro de la función docente no está basado solo en la afiliación institucional de los autores sino, sobre todo, en las descripciones que se hacen en los trabajos de los profesores que colaboran en las investigaciones. En estos artículos participan profesores de todos niveles educativos, dándose la casi totalidad de posibles combinaciones entre ellos y, en pocos casos, con algún otro profesional. La tabla 3 contiene las categorías que reúnen más de un trabajo.

Tabla 3.

Categorías principales de la tipología del profesorado investigador

\begin{tabular}{|l|c|c|c|c|}
\hline \multicolumn{1}{|c|}{$\begin{array}{c}\text { TIPOLOGÍA } \\
\text { DOCENTE }\end{array}$} & $\begin{array}{c}\text { ARTÍCULOS CON } \\
\text { 1 INVESTIGADOR }\end{array}$ & $\begin{array}{c}\text { ARTÍCULOS CON } \\
\text { 2 INVESTIGADORES }\end{array}$ & $\begin{array}{c}\text { ARTÍCULOS CON } \\
\text { 3 o MÁS INVESTIGADORES }\end{array}$ & $\begin{array}{c}\text { TOTAL } \\
\text { ARTÍCULOS }\end{array}$ \\
\hline Prof. DCE & 3 & 1 & 8 & 13 \\
\hline $\begin{array}{l}\text { Prof. DCE y } \\
\text { Prof. Secund. }\end{array}$ & 5 & 5 & 5 \\
\hline $\begin{array}{l}\text { Prof. DCE, otros Prof. } \\
\text { Univ. y Prof. Secund. }\end{array}$ & & & 2 & 2 \\
\hline $\begin{array}{l}\text { Prof. DCE, Prof. Sec. } \\
\text { y Prof. Prim. }\end{array}$ & & 2 & 3 & 2 \\
\hline $\begin{array}{l}\text { Otros Prof. Univ. y } \\
\text { Prof. Secundaria }\end{array}$ & 3 & 1 & & 7 \\
\hline Prof. Secundaria & & & & 2 \\
\hline
\end{tabular}


En la investigación-acción española realizada en Didáctica de las Ciencias Experimentales ha predominado la colaboración que vincula a los profesores de dicho ámbito científico-didáctico y a los de Enseñanza Secundaria. En una segunda posición se sitúa la realizada conjuntamente por profesores de Enseñanza secundaria, seguida por la efectuada de forma participativa entre ambos tipos de profesores mencionados y otros profesores de universidad. Las tres categorías reúnen un 53,2\% del total y el conjunto de las presentadas, en la tabla 3, un $70,2 \%$.

$\mathrm{Al}$ descender en el análisis al número y al tipo de roles de los profesores, encontramos que en un $38,3 \%$ de los artículos no se especifican los roles o se hace de forma incompleta, en un 21,3\% de ellos existe un solo rol, que es el de profesor investigador de su acción docente; en un 36,2\% hay dos roles, siendo el predominante, en un $27,7 \%$ de los artículos, el rol previo y el de profesor investigador facilitador, con esa formulación u otras próximas, y, en el otro 8,5\%, el mencionado rol de profesor investigador de su acción docente unido con el de investigador externo (6,4\%), y el primero de estos dos últimos junto con el de asesor de un CEP (2,1\%). Finalmente, en un 4,2\% de los trabajos identificamos tres roles: profesor investigador de su acción, profesor investigador facilitador y maestro-asesor de un CPR $(2,1 \%)$ y los dos primeros con el de profesor colaborador $(2,1 \%)$.

\section{Incidencia de la investigación-acción en la práctica docente y en el desarrollo profesional}

La investigación-acción española ha tenido una incidencia positiva en las prácticas dirigidas a la formación inicial del profesorado, en las orientadas a la formación y el desarrollo profesional del profesorado en activo, así como en aquellas comprometidas con la enseńanza y el aprendizaje de las Ciencias Experimentales y de su propia didáctica. El ejercicio de esta ha posibilitado el desarrollo profesional de los profesores-investigadores mediante el aprendizaje y el desarrollo de las habilidades propias de ese enfoque, a través de la innovación curricular y de la adquisición de conocimientos.

Al no ser posible, por los límites de espacio del presente trabajo, especificar esas incidencias en el conjunto de las investigaciones, presentamos, en el cuadro 4, una síntesis de algunas de las más significativas de un pequeño número de ellas, las cuales sustentan la afirmación previa. 
Cuadro 4.

Incidencia de la investigación-acción en la práctica y en el desarrollo profesional

- El desarrollo curricular relativo al medio ambiente y la salud incide positivamente en el trabajo en grupo de los alumnos, en el clima psicosocial del aula y en la satisfacción de los implicados. Se favorece el aprendizaje de conocimientos de los estudiantes, su capacidad de expresión y las relaciones socio-afectivas, entre otros aspectos. El profesorado se desarrolla profesionalmente a través de la ampliación de conocimientos, de la mejora de sus habilidades y de su incorporación en una comunidad académica de innovación e investigación educativa.

- A través del conocimiento del estado en el que se encuentra el contenido de la reflexión de una profesora de ciencias y de su evolución hacia una mayor complejidad, que se producen desde la investigación-acción, se incide en su formación y su desarrollo profesional y, simultáneamente, en su práctica docente. Además, la comprensión del proceso puesto en marcha en esa profesora que se ha comprometido con la innovación curricular y la mejora profesional, y, en particular, la de los obstáculos que impiden dicha complejidad en la reflexión, supone desarrollo profesional para todos los profesores participantes en la investigación.

- La realización de diseños de prácticas de laboratorio atractivas y motivadoras para los alumnos de Secundaria ha contribuido a fomentar la innovación en el aula, a potenciar el número de horas dedicadas al trabajo en el laboratorio, a instruir a los alumnos en el método científico mediante una metodología práctica, a potenciar el trabajo en equipo entre los profesores del Departamento de Ciencias y a promover su formación mediante la investigación y el fomento de los aspectos prácticos relacionados con sus áreas de conocimiento.

- Contribución a la mejora de la eficacia y eficiencia en la integración de la educación ambiental en los centros educativos, incidiendo en el desarrollo profesional de los profesores participantes. Uno de los logros más importantes ha sido la ambientalización del centro. Asimismo, destaca la motivación de los alumnos, que lleva consigo la adquisición de hábitos y actitudes acordes con los compromisos. Además, entre los múltiples indicadores de calidad, señalamos la elaboración de materiales, creación de una revista y de una página web sobre la experiencia, realización de publicaciones y diseńo de un plan formativo para seguir avanzando, entre otros.

- Desarrollo del currículum de ciencias de la formación inicial de maestros mediante una unidad didáctica centrada en la energía y en la utilización de la metodología de resolución de problemas como investigación. Se produce una mejora relevante en los aprendizajes de los procedimientos de dicha metodología y sobre los contenidos científicos relativos a la energía. Se contribuye al conocimiento de Didáctica de las Ciencias Experimentales al confirmar la MRPI en el marco del Espacio Europeo de la Educación Superior.

\section{MODO DE CONCLUSIÓN}

El presente trabajo aporta, por una parte, una revisión clarificadora de la investigación-acción y, por otra, una perspectiva de su desarrollo en Didáctica de las Ciencias Experimentales a través de las revistas españolas de educación. Mediante la identificación de los artículos en las bases de datos especializas y el análisis de estos, ha quedado configurado un perfil personal, social y cognitivo de dicha línea de investigación.

Se ha identificado un desarrollo muy incipiente de dicho enfoque de investigación en España, a pesar de que los autores de otros países han hecho una difusión importante de sus trabajos en el nuestro. Este hallazgo confirma, en mayor grado, que la investigación del profesor de ciencias se encuentra en su infancia. No obstante, la investigación-acción realizada ha tenido una incidencia positiva en la enseñanza y el aprendizaje de las Ciencias Experimentales y de su didáctica, en la formación de sus profesores y en su desarrollo profesional.

Nuestro trabajo pone de relieve la necesidad de potenciar esta modalidad de investigación en el área de Didáctica de las Ciencias, en orden a lograr una mejora en sus procesos de enseńanza y aprendizaje, una resolución de las cuestiones problemáticas que se detecten en sus prácticas y un afrontamiento de los retos que la sociedad actual plantea al sistema educativo. Esta investigación se ha de incrementar en todas las líneas de investigación habituales en Didáctica de las Ciencias Experimentales. Junto con los 
escasos grupos de investigación identificados hay que impulsar la creación de nuevas redes, en las que puedan integrarse profesores de Ciencias Experimentales de los distintos niveles del sistema educativo y de Didáctica de las Ciencias Experimentales.

Una vía para impulsar dicha investigación seria incorporarla de forma generalizada, como una estrategia metodológica, en los prácticum de los planes de estudio de la formación de maestros y profesores de Enseñanza Secundaria, estableciendo una interrelación entre ambos que conduzca a estudios de casos de amplio alcance.

Por último, ante la ausencia detectada del paradigma participativo en la investigación-acción española, postulamos la conveniencia incorporarlo a ella, por ser un enfoque valioso tanto para la Didáctica de la Ciencias Experimentales como para la Pedagogía.

\section{REFERENCIAS BIBLIOGRÁFICAS}

Abell, S. K. y Lederman, N. G. (2008). Handbook of research on science education. New York: Routledge.

Anta, C. (2008). Análisis bibliométrico de la investigación educativa divulgada en publicaciones periódicas españolas entre 1990-2002. Revista Electrónica de Investigación Educativa, 10 (1), pp. 1-17.

Anta, C. y Pérez, J. M. (2007, noviembre, 21-23). La producción cientifica sobre Didáctica de las Ciencias. IV Congreso Comunicación Social de la Ciencia. Madrid: CSIC.

Benarroch, A. (2010). La investigación en Didáctica de las Ciencias Experimentales en las etapas educativas de Infantil y Primaria. En A.M. Abril y A. Quesada (eds.). XXIV Encuentro de Didáctica de las Ciencias Experimentales (pp. 32-52). Baeza, Jaén: Servicio de Publicaciones de la Universidad de Jaén.

BradFord, S.C. (1934). Sources of information on specific subjects. Engineering, 137, pp. 85-86.

Carr, W. y Kemmis, S. (1988). Teoría critica de la enseñanza. Barcelona: Martínez-Roca.

Colás, M. P. (2007). La investigación acción y la generación de conocimiento educativo. En M. Campillo y A. Zaplana (coords.). Investigación, educación y desarrollo profesional. Murcia: Diego Marín, pp. 49-75.

COMISIÓN DE LAS COMUNIDADES EUROPEAS y CONSEJO De EUROpa (1998). Tesauro Europeo de la Educación. Luxemburgo: Autor.

Corey, S. (1953). Action research to improve school practices. New York: Columbia University, Teachers College.

De La Gandara, M. (1992). La investigación en enseñanza de las ciencias en España. Revista Interuniversitaria de Formación del profesorado, 14, pp. 19-26.

De Pro, A. (1998). ¿Qué investigamos?, ¿cómo lo hacemos?, ¿̨a qué conclusiones llegamos?: tres preguntas que hacen pensar. En C. Martínez y S. García (eds.). La Didáctica de las Ciencias. Tendencias actuales. A Coruña: Universidade da Coruña, pp. 19-43.

De Pro, A. (2009) ¿Qué investigamos en Didáctica de las Ciencias Experimentales en nuestro contexto educativo? Investigación en la Escuela, 69, pp. 45-59.

Elliott, J. (1993). El cambio educativo desde la investigación-acción. Madrid: Morata.

Elórtegui, N., Fernández, J. y Medina, M. (2002). Estudio bibliométrico sobre la investigación en Didáctica de las Ciencias de la Naturaleza. Alambique, 34, pp. 82-83.

Furio, C.J. (1994). Tendencias actuales en la formación del profesorado de ciencias. Enseñanza de las Ciencias, 12(2), pp. 188-199.

Furió, C.J. y Martínez, F.S. (1985). Investigación española en la enseñanza de las ciencias (19761982). Primeros resultados. Enseñanza de las Ciencias, (extra), p. 6. 
García Barros, S. (2008). La formación del profesorado de educación infantil. En M.R. Jiménez (ed.). Actas de los XXIII Encuentros de Didáctica de las Ciencias Experimentales. Almería: Universidad de Almería, pp. 246-255.

García Carmona, A. (2009). La investigación acción en la enseñanza de la Física: un escenario idóneo para la formación y el desarrollo profesional del profesorado. Latin-American Journal of Physics Education, 3(2), pp. 288-394.

Gil, D. (1994). Diez años de investigación en Didáctica de las Ciencias: realizaciones y perspectivas. Enseñanza de las Ciencias, 12(2), pp. 154-164.

Guba, E. G. y Lincoln, Y.S. (2005). Paradigmatic controversies, contradictions and emerging confluences. En N.K. Denzin y Y.S. Lincoln (eds.). The Sage handbook of qualitative research (3 $\left.{ }^{\text {rd }} \mathrm{ed}.\right)$. Thousand Oaks, CA: Sage, pp. 191-215.

Kemmis, S. y Mctaggart, R. (1992). Cómo planificar la investigación-acción. Barcelona: Laertes.

LeE, M., Wu, y Tsai, C. (2009). Research trends in Science Education from 2003 to 2007: A content analysis of publications in selected journals. International Journal of Science Education, 15(1), pp. 1999-2020. http://dx.doi.org/10.1080/09500690802314876

Mata, M. y Anta, C. (1985). Evolución y nuevas tendencias en los trabajos sobre Didáctica de las Ciencias Experimentales. Enseñanza de las Ciencias, (extra), p. 4

Mata, M. y Méndez, A. (1985). La renovación didáctica en las Ciencias experimentales. Estudio bibliométrico. Enseñanza de las Ciencias, 3(1), pp. 3-10.

Maz, A., Torralvo, M., Vallejo, M., Fernández-Cano, A. y Rico, L. (2009). La educación matemática en la revista Enseñanza de las Ciencias: 1983-2006. Enseñanza de las Ciencias, 27(2), pp. 185-194.

Moreira, M.A. (1994). Diez años de la revista Enseñanza de las Ciencias: de una ilusión a una realidad. Enseñanza de las Ciencias, 12(2), pp. 147-153.

Morín, E. (1998). Introducción al pensamiento complejo. Barcelona: Gedisa.

Oliva, J.M. (2006). Reseña de 'Seminario Internacional sobre «el estado actual de la investigación en enseñanza de las ciencias'» de A. F. Cachapuz, B. Lopes, F. Paixao, J. F. Praia y C. Guerra. Revista Eureka sobre Enseñanza y Divulgación de las Ciencias, 3(1), pp. 167-171.

Oliva, J. M. (2010). La educación secundaria como foco de atención en las publicaciones de didáctica de las ciencias de revistas de nuestro entorno. En A.M. Abril y A. Quesada (eds.). XXIV Encuentro de Didáctica de las Ciencias Experimentales. Baeza, Jaén: Servicio de Publicaciones de la Universidad de Jaén, pp.53-66.

Palacios, C. y Ansoleaga, D. (1993). Diez años de investigación e innovación en enseñanza de las ciencias desde el CIDE. Enseñanza de las Ciencias, (número extra), pp.113-114.

Peiró, J. M. (1981). «Colegios invisibles» en Psicología. En H. Carpintero y J. M. Peiró (dirs.), Psicología contemporánea. Valencia: Alfaplus, pp. 53-78.

Price, D. y Beaver, D. (1966). Collaboration in an invisible college. American Psychologist, 21(11), pp. 1011-1018.

http://dx.doi.org/10.1037/h0024051

Reason, P. y Bradbury, H. (2005). Preface. En P. Reason y H. Bradbury (eds.). Handbook of action research. Participative inquiry and practice (pp. xxiii-xxxi). London: Sage.

Roth, K.J. (2008). Science teachers as researchers. En S.K. Abell y N. G. Lederman (eds.). Handbook of research on science education. New York: Routledge, pp. 1205-1259.

SÁEz, M.J. (1987). La investigación-acción y la formación del profesorado. Investigación en la Escuela, 2, pp.15-20. 
Schwandt, T.A. (1994). Constructivist, interpretivist approaches to human Inquiry. In N. K. Denzin y Y. S. Lincoln (eds.). Handbook of qualitative research. Thousand Oaks, CA: Sage, pp. 118-137.

Schön, D.A. (2002). La formación de profesionales reflexivos. Hacia un nuevo diseño de la enseñanza y el aprendizaje en las profesiones. Barcelona: Paidós/MEC.

Shafer, L. (2000). Teacher research continuum chart. Disponible en: http://www.gse.gmu.edu/research/tr/trprofessional.shtml. (Última consulta, 13 de septiembre de 2004).

Stenhouse, L. (1985, mayo/agosto). El profesor como tema de investigación y desarrollo. Revista de Educación, 277, pp. 43-53.

Stenhouse, L. (1984). Investigación y desarrollo del currículum. Madrid: Morata.

TsaI, C. y Wen, M.L. (2005). Research and trends in science education from 1998 to 2002: a content analysis of publication in selected journals. International Journal of Science Education, 27(1), pp. $3-14$.

http://dx.doi.org/10.1080/0950069042000243727

Zeichner, K. (2005). Educational Action Research. En P. Reason y H. Bradbury (eds.). Handbook of Action Research. Participative inquiry and practice. London: Sage, pp. 273-283.

Zeichner, K. y Noffke, S. (2001). Practitioner research. En V. Richardson (ed.). Handbook of research on teaching. Washington, DC: AERA, pp. 298-330. 


\title{
Action research in the field of science education: a view from spanish journals on education
}

\author{
María Jesús Romera Iruela \\ Departamento de Teoría e Historia de la Educación, Universidad Complutense, Madrid \\ mjromera@edu.ucm.es
}

This article examines the level of development of action research and its impact on Science Education. This approach needs to take top priority in the work of science education professionals, as it leads to valuable change and continuous improvement to teaching practice, thus helping to bridge the gap between theory and practice. The aim of the research is to ascertain the presence of action research in Science Education articles published in Spanish journals of education. The article establishes two levels of analysis, one taking an overview of all the articles dealing with the topic of study and another focusing solely on Spanish articles adopting this particular research approach.

The questions formulated in the study include the following:

To what extent have foreign studies been disseminated in Spanish journals? What research groups can be identified? What are the main paradigms guiding action research? Which are the most researched topics? What are the roles of Spanish researchers within the teaching function? What are the repercussions of action research on teaching practice and professional development?

This article formulates a conceptual framework for action research in education and for this study in particular. The action research articles were identified by following a sequential process of consulting the main specialist education databases, together with further information obtained from multidisciplinary journal databases. These databases were queried using a combination of natural and documentary languages. Once the documents had been retrieved, the content analysis technique was applied on the various aspects addressed by the topic of study and the data obtained were subsequently analysed, interpreted and discussed.

The research provides knowledge of the personal, social and cognitive dimensions of Spanish action research in Science Education, which contributes to the understanding of the epistemological and professional identity of this area of science. It was observed that this approach is still largely underdeveloped in Spain, despite a noticeable presence of publications in this country of studies by authors based abroad. This finding confirms to an even greater degree that research conducted by science teachers is still in its infancy. Action research features in ten of the customary Science Education research lines practised today. Its contribution to teacher training has therefore consisted of proposals aimed at changing attitudes and practices towards constructivist approaches; equipping teachers with the skills for designing curricular projects and to do research on their own actions in the classroom, and at developing their practice based on the model of teaching as a reflective profession. The majority of these proposals are aimed at practising teachers. In terms of the teaching role carried out by Spanish action researchers who have published articles in this scientific field, there is a predominance of links between Experimental Science Education and Secondary Education teachers. It was found that Spanish action research has had a positive impact on practices focused both on pre-service teacher training and on in-service teacher training and professional development, as well as on those centred on teaching and learning in Experimental Sciences and Science Education. The article concludes by pointing out the need to promote the creation of new networks involving science teachers from different levels of the educational system as well as Science Education teachers. One way of promoting this research approach would be to incorporate it as a widespread methodological strategy in the Practicum of pre-school, primary and secondary education teacher training study programmes. 